\title{
Density and Extension of Differentiable Functions on Metric Measure Spaces
}

https://doi.org/10.1515/agms-2020-0130

Received June 5, 2021; accepted November 22, 2021

Abstract: We consider vector valued mappings defined on metric measure spaces with a measurable differentiable structure and study both approximations by nicer mappings and regular extensions of the given mappings when defined on closed subsets. Therefore, we propose a first approach to these problems, largely studied on Euclidean and Banach spaces during the last century, for first order differentiable functions defined on these metric measure spaces.

Keywords: Smooth extensions; smooth approximations; metric measure spaces; measurable differentiable structures; Lipschitz mappings

MSC: 6A24, 28A15, 54C20, 54E35, 54E99.

\section{Introduction}

In this paper we deal with problems about density of regular mappings and regular extensions of regular mappings on metric measure spaces endowed with a measurable differentiable structure (MDS for short) in the sense of J. Cheeger [6]. We present here a first order differentiability study on these questions following the steps already given in Euclidean spaces as well as in finite dimensional Banach spaces on the same questions.

In any context it is natural to wonder whether a function can be approximated by another one with better properties. This is one of our goals for functions defined on metric measure spaces with MDS. More precisely, we will study the existence of differentiable (differentiable and Lipschitz) approximations for continuous (continuous and Lipschitz) vector valued mappings. There exists a large literature on this subject, mainly given on linear spaces, developed during the last century where a whole collection of related problems are considered, the interested reader may check, for instance, $[5,7,11,27]$ and references therein.

The extension problem for mappings defined on closed subsets of finite dimensional Banach spaces has been extensively studied. The first one in dealing with this problem was $\mathrm{H}$. Whitney [29, 30], who characterized functions defined on closed subsets of the real line that can be extended to the whole real line as functions of $C^{k}$ class. Then, G. Gleaser [12] studied the same problem for higher dimensions and $C^{1}$ extensions and, finally, C. Fefferman [9, 10], in a series of papers, completed the program for finite dimensional spaces and functions defined on compact subsets of them with $C^{k}$ class extensions.

\footnotetext{
${ }^{\star}$ Corresponding Author: Rafael Espinola Garcıa: Department of Mathematical Analysis - IMUS, University of Seville, Sevilla, Spain, E-mail: espinola@us.es

Luis Sánchez González ${ }^{1}$ : Departamento de Ingeniería Matemática, Facultad de CC. Físicas y Matemáticas, Universidad de Concepción, Concepción, Chile

Note from the corresponding author: my coauthor in this paper, Luis Sánchez González, passed away some time ago in an accident while hiking in the mountains. The paper in its current state is not much different to how it was when he passed away. We were working at that moment on solving some final technical details. Some of them have been solved now and that is why it has come the time to see our work published.
} 
In Banach spaces of infinite dimension the situation of the problem is far less well understood although there has been a large collection of publications on the topic, see, for instance, $[1-3,15,16,19,20,27]$. Here the variety of regularity notions and the kind of spaces under consideration vary upon the goals of the authors.

Metric measure spaces endowed with a measurable differentiable structure (MDS) in the sense of J. Cheeger were introduced in [6]. These spaces have called the attention of many authors since then and has helped to develop a first order differential analysis on certain metric spaces. The interested reader may check $[4,6,13,14,22,23]$ and references therein. These spaces will allow us for a first order differentiability notion for the functions we are going to deal with and, of course, the notion of derivative we will deal with is that of derivative with respect to a chart given by J. Cheeger [6] and S. Keith [22].

In Section 2 we give the precise definition of a metric measure space with a MDS, this is basically the same notion that we find in $[6,22]$. In Section 3 we study the problem of the density of differentiable functions when defined from a metric measure space into a Banach space. We consider first the case of approximating vector valued continuous mappings by differentiable almost everywhere ones and, then, we study the same problem for continuous and Lipschitz functions which will be approximated by differentiable almost everywhere and Lipschitz ones (see Theorems 3.1 and 3.7). The condition of the metric space to be doubling will also be important for the density results we obtain. Some of these results will be applied in Section 4, where we deal with the problem of the differentiable extension of differentiable functions defined from a metric measure space into a Banach space. We prove here a number of partial results on the pursuit of, under adequate conditions, proving that every differentiable almost everywhere, or differentiable almost everywhere and Lipschitz, mapping can be extended as differentiable almost everywhere, or, respectively, differentiable almost everywhere and Lipschitz mappings, to the whole space (see Theorems 4.5 and 4.8).

\section{Measurable Differentiable Structures}

In this section we describe the notion of metric measure space with a measurable differentiable structure introduced by J. Cheeger [6] and S. Keith [22], as well as the associated notions as that of differentiable almost everywhere mapping. Metric measure spaces with MDS were also called Lipschitz differentiability spaces in [4]. The interested reader may also check the survey [23] to learn more about them.

Given a metric space $X$, the set $\operatorname{LIP}(X)$ denotes the set of all Lipschitz real functions on $X$. We give next the precise definition of a metric measure space that admits a measurable differentiable structure.

Definition 2.1. (Cheeger, Keith). Let $(X, d, \mu)$ be a metric measure space, and let $\mathscr{C} \subset \operatorname{LIP}(X)$ be a vector space of functions.

(A) A pair $(Y, \mathbf{y})$ is a $\mathscr{C}$-chart if $Y \subset X$ is a measurable subset with $\mu(Y)>0$ and $\mathbf{y}=\left(\mathbf{y}_{\mathbf{1}}, \ldots, \mathbf{y}_{\mathbf{k}}\right): X \rightarrow \mathbb{R}^{k}$ is a function for some $k \in \mathbb{N} \cup\{0\}$, called coordinates on $Y$, where $y_{i} \in \mathscr{C}$ for every $1 \leq i \leq k$.

(B) The metric measure space $(X, d, \mu)$ has a $\mathscr{C}$-measurable differentiable structure $(\mathscr{C}$-MDS, for short) if there is a countable collection of $\mathscr{C}$-charts $\left\{\left(X_{\alpha}, \mathbf{y}^{\alpha}\right)\right\}_{\alpha \in \mathscr{A}}$, which is called a $\mathscr{C}$-atlas of $X$, with coordinates $\mathbf{y}^{\alpha}: X \rightarrow \mathbb{R}^{k(\alpha)}$, so that

$$
\mu\left(X \backslash \bigcup_{\alpha \in \mathscr{A}} X_{\alpha}\right)=0,
$$

$k=\sup _{\alpha} k(\alpha)<\infty$, and for every $f \in \mathscr{C}$ and chart $\left(X_{\alpha}, \mathbf{y}^{\alpha}\right)$ there exists a unique (up to a set of zero measure) measurable function $d f^{\alpha}: X_{\alpha} \rightarrow \mathbb{R}^{k(\alpha)}$ such that

$$
\limsup _{z \rightarrow x} \frac{\left|f(z)-f(x)-d f^{\alpha}(x) \cdot\left(\mathbf{y}^{\alpha}(z)-\mathbf{y}^{\alpha}(x)\right)\right|}{d(z, x)}=0
$$

for $\mu$-a.e. $x \in X_{\alpha}$. Moreover, such a structure is called $k$-dimensional and it is non-degenerate if $k(\alpha) \geq 1$ for all $\alpha \in \mathscr{A}$. We will assume we always work with non-degenerate MDS. 
(C) We say that the pair $(Y, \mathbf{y})$ is a chart, the countable collection $\left\{\left(X_{\alpha}, \mathbf{y}^{\alpha}\right)\right\}_{\alpha \in \mathscr{A}}$ is an atlas of $X$ and the metric measure space $(X, d, \mu)$ has a measurable differentiable structure (MDS, for short) whenever $(Y, \mathbf{y})$ is a $\operatorname{LIP}(X)$-chart, $\left\{\left(X_{\alpha}, \mathbf{y}^{\alpha}\right)\right\}_{\alpha \in \mathscr{A}}$ is a $\operatorname{LIP}(X)$-atlas of $X$ and $(X, d, \mu)$ has a $\operatorname{LIP}(X)$-MDS.

In references $[6,22,23]$ the reader may find examples of metric spaces that can be endowed with a MDS and main facts on them. Next we define what we understand by differentiability at a point of mappings acting on these structures.

Definition 2.2. Let $X$ be a metric space with a $\mathscr{C}$-MDS and let $\left(X_{\alpha}, \mathbf{y}^{\alpha}\right)$ be a $\mathscr{C}$-chart of $X$ and $V$ a Banach space.

(a) A function $f: X \rightarrow \mathbb{R}$ is $\mathscr{C}$-differentiable at $x \in X_{\alpha}$ (just differentiable if $\mathscr{C}=\operatorname{LIP}(X)$ ), with respect to $\left(X_{\alpha}, \mathbf{y}^{\alpha}\right)$, if there is a linear function $d f^{\alpha}(x): \mathbb{R}^{k(\alpha)} \rightarrow \mathbb{R}$ such that

$$
\limsup _{z \rightarrow x} \frac{\left|f(z)-f(x)-d f^{\alpha}(x) \circ\left(\mathbf{y}^{\alpha}(z)-\mathbf{y}^{\alpha}(x)\right)\right|}{d(z, x)}=0 .
$$

(b) A mapping $f: X \rightarrow V$ is $\mathscr{C}$-differentiable at $x \in X_{\alpha}$ (just differentiable if $\mathscr{C}=\operatorname{LIP}(X)$ ), with respect to $\left(X_{\alpha}, \mathbf{y}^{\alpha}\right)$, if there is a linear function $d f^{\alpha}(x): \mathbb{R}^{k(\alpha)} \rightarrow V$ such that

$$
\limsup _{z \rightarrow x} \frac{\left\|f(z)-f(x)-d f^{\alpha}(x) \circ\left(\mathbf{y}^{\alpha}(z)-\mathbf{y}^{\alpha}(x)\right)\right\|}{d(z, x)}=0 .
$$

Finally, we can state what we mean by differentiability almost everywhere of a mapping defined on metric measure spaces with a MDS.

Definition 2.3. Let $X$ be a metric space with a $\mathscr{C}$-MDS and let $\left(X_{\alpha}, \mathbf{y}^{\alpha}\right)$ be a $\mathscr{C}$-chart of $X$ and $V$ a Banach space. A mapping $f: X \rightarrow V$ is $\mathscr{C}$-differentiable almost everywhere (just differentiable almost everywhere if $\mathscr{C}=\operatorname{LIP}(X))$ with respect to the $\mathscr{C}$-atlas $\left\{\left(X_{\alpha}, \mathbf{y}^{\alpha}\right)\right\}_{\alpha \in \mathscr{A}}$ if there is a collection $\left\{\frac{d f}{y_{m}^{\alpha}}: X_{\alpha} \rightarrow V\right\}_{\alpha \in \mathscr{A}, 1 \leq m \leq k(\alpha)}$ of measurable functions uniquely determined (up to a set of zero measure), such that for almost every $x \in X_{\alpha}$

$$
\limsup _{z \rightarrow x} \frac{\left\|f(z)-f(x)-\sum_{m=1}^{k(\alpha)} \frac{d f}{d y_{m}^{\alpha}}(x)\left(y_{m}^{\alpha}(z)-y_{m}^{\alpha}(x)\right)\right\|}{d(z, x)}=0 .
$$

Remark 2.4. Notice that if we are in the case $\mathscr{C}=\operatorname{LIP}(X)$, then real-valued Lipschitz functions will be differentiable almost everywhere by definition. This fact will be used at several instances regarding the distance function. Also, mappings in the chart are differentiable almost everywhere and Lipschitz.

\section{Density of differentiable functions}

In this section we study properties of density of differentiable almost everywhere mappings. We begin by showing that any continuous mapping can be approximated by such mappings. We will achieve our goal by applying standard techniques on partitions of unity. To make the exposition easier, we will work with MDS instead of $\mathscr{C}$-MDS although some comments will be added on this regard.

Theorem 3.1. Let $(X, d, \mu)$ be a separable metric measure space with a MDS and let $V$ be a Banach space. Then, for every continuous mapping $f: X \rightarrow V$ and every $\varepsilon>0$, there is a differentiable almost everywhere mapping $g: X \rightarrow V$ such that

$$
\|f(x)-g(x)\|<\varepsilon \quad \text { for all } x \in X .
$$

Proof. Since $X$ is separable and $f$ is continuous, there exists a covering $\left\{B\left(x_{n}, \frac{r_{n}}{2}\right)\right\}_{n=1}^{\infty}$ of $X$ by open balls, such that

$$
|| f(x)-f\left(x_{n}\right) \|<\varepsilon
$$


for every $x \in B\left(x_{n}, r_{n}\right)$.

We claim that there is a differentiable almost everywhere (and Lipschitz) partition of unity subordinated to the covering $\left\{B\left(x_{n}, r_{n}\right)\right\}_{n=1}^{\infty}$ of $X$. Indeed, by a standard procedure, we can take $C^{1}$ smooth and Lipschitz functions $\theta_{n}: \mathbb{R} \rightarrow[0,1]$ such that $\theta_{n}(t)=1$ whenever $t \leq \frac{r_{n}}{2}, \theta_{n}(t)=0$ whenever $t \geq r_{n}$ and $\operatorname{Lip}\left(\theta_{n}\right) \leq 3 / r_{n}$. Let us define $\psi_{n}: X \rightarrow[0,1]$ as

$$
\psi_{n}(x)=\theta_{n}\left(d\left(x, x_{n}\right)\right) \text { for } x \in X .
$$

Functions $\psi_{n}$ are differentiable almost everywhere (and Lipschitz), $\psi_{n}(x)=1$ on $B\left(x_{n}, \frac{r_{n}}{2}\right)$ and $\psi_{n}(x)=0$ on $X \backslash B\left(x_{n}, r_{n}\right)$. Let us define now the functions $\varphi_{n}: X \rightarrow[0,1]$ as

$$
\varphi_{n}(x)=\psi_{n}(x) \prod_{k=1}^{n-1}\left(1-\psi_{k}(x)\right) .
$$

The functions $\varphi_{n}$ are differentiable almost everywhere (and Lipschitz). Moreover, it is easy to see that $\sum_{n=1}^{\infty} \varphi_{n} \equiv$ 1 , for each $x \in X$ there is a neighborhood of $x$ where all but a finite number of functions $\varphi_{n}$ are 0 , and

$$
\operatorname{supp} \varphi_{n}=\overline{\left\{x \in X: \varphi_{n}(x) \neq 0\right\}} \subset B\left(x_{n}, r_{n}\right) .
$$

Consider now the function $g: X \rightarrow V$ defined as

$$
g(x)=\sum_{n=1}^{\infty} \varphi_{n}(x) f\left(x_{n}\right) .
$$

This function is differentiable almost everywhere because the family $\left\{\varphi_{n}\right\}_{n=1}^{\infty}$ is locally finite and differentiable almost everywhere. Let us see that $g$ uniformly approximates $f$. For every $x \in X$

$$
\|f(x)-g(x)\| \leq \sum_{n=1}^{\infty} \varphi_{n}(x)\left\|f(x)-f\left(x_{n}\right)\right\|<\varepsilon,
$$

where the last inequality is a consequence of the fact that $\left\|f(x)-f\left(x_{n}\right)\right\|<\varepsilon$ whenever $\varphi_{n}(x) \neq 0$.

\section{Remark 3.2.}

(i) Let us note that if $\mathscr{C} \subset \operatorname{LIP}(X)$ is a vector space of functions such that $(X, d, \mu)$ has a $\mathscr{C}$-MDS, the above theorem holds whenever the functions $x \mapsto d\left(x, x_{0}\right)$ belong to $\mathscr{C}$ for all $x_{0} \in X$.

(ii) Furthermore, if the functions $x \mapsto d\left(x, x_{0}\right)$ are differentiable everywhere but $x_{0}$, then the approximation mapping is differentiable everywhere on $X$ (assuming $X=\cup_{\alpha} X_{\alpha}$ ).

Now we seek approximations by mappings that are differentiable almost everywhere and Lipschitz. For that we will need the extra hypothesis of working with doubling metric spaces which, in particular, are separable.

Definition 3.3. A metric space $(X, d)$ is said to be doubling if there is a constant $C$ such that for each $r>0$, every ball contained in $X$ with radius $r$ can be covered by at most $C$ balls of radius $r / 2$. The doubling constant $\lambda(X)$ if the infimum over all constants $C$ satisfying the doubling condition.

Doubling metric spaces have played a major role in the recent theory of analysis on metric spaces, as reader may check in references $[6,22,23]$ or the monograph by J. Heinonen [13]. Notice also that if $(X, d)$ is doubling, then for each $r>0$, every ball contained in $X$ with radius $r$ can be covered by at most $\lambda(X)^{n}$ balls of radius $r / 2^{n}$.

The following theorem is needed to prove the next density result.

Theorem 3.4 ([24]). There exists a universal constant $C>0$ such that for every doubling metric space $(X, d)$, for every $Y \supset X$ and $V$ Banach spaces, and for every Lipschitz mapping $f: X \rightarrow V$, there exists a Lipschitz extension $F: Y \rightarrow V$ such that

$$
\operatorname{Lip}(F) \leq C \lambda(X) \operatorname{Lip}(f) .
$$


Another tool that we will use in this section is the concept of functions that locally depend on finitely many coordinates. This notion was first defined on Banach spaces with Schauder basis using the coordinate functionals [25]. Later, a generalization of this notion was considered by some authors using arbitrary continuous linear functionals, see, for instance, $[8,18,28]$.

Definition 3.5. Let $Y$ and $Z$ be Banach spaces, $M \subset Y^{\star}$ and $G: Y \rightarrow Z$. We say that $\mathrm{G}$ locally depends on finitely many coordinates from $M$ ( $L F C-M$, for short) if for each $x \in Y$ there are a neighborhood $U$ of $x$, a finite subset $\left\{f_{1}, \ldots, f_{n}\right\} \subset M$ and a mapping $H: \mathbb{R}^{n} \rightarrow Z$ such that $G(x)=H\left(f_{1}(x), \ldots, f_{n}(x)\right)$ for all $x \in U$.

A simple example is the sup norm on $c_{0}$, which is $\operatorname{LFC}-\left\{e_{n}^{\star}\right\}_{n=1}^{\infty}$ away from the origin (where $\left\{e_{n}^{\star}\right\}_{n=1}^{\infty}$ are the coordinate functionals in $c_{0}$ ).

Lemma 3.6. Let $(X, d, \mu)$ be a metric measure space with a MDS and let $V$ be a Banach space. Let $\Phi: X \rightarrow c_{0}$ be a mapping whose coordinate functions $e_{n}^{\star} \circ \Phi: X \rightarrow \mathbb{R}$ are differentiable almost everywhere, and $G: c_{0} \rightarrow V$ $a L F C-\left\{e_{n}^{\star}\right\}_{n=1}^{\infty}$ and $C^{1}$ smooth map. Then, the mapping $G \circ \Phi: X \rightarrow V$ is differentiable almost everywhere.

Proof. The coordinate functions $e_{n}^{\star} \circ \Phi: X \rightarrow \mathbb{R}$ are differentiable almost everywhere. Then, for every $x \in$ $\left\{y \in X: e_{n}^{\star} \circ \Phi\right.$ is differentiable at $y$ for all $\left.n \geq 1\right\}$, there is a neighborhood $U$ of $x$, a finite subset $\left\{e_{1}^{\star}, \ldots, e_{n}^{\star}\right\}$ of functionals and a $C^{1}$ smooth mapping $H: \mathbb{R}^{n} \rightarrow V$ such that $G(y)=H\left(e_{1}^{\star}(y), \ldots, e_{n}^{\star}(y)\right)$ for all $y \in \Phi(U)$ (see [27]). Thus, $G \circ \Phi(y)=H\left(e_{1}^{\star} \circ \Phi(y), \ldots, e_{n}^{\star} \circ \Phi(y)\right)$ for all $y \in U$, which is differentiable at $x$. Since the set $\left\{x \in X: e_{n}^{\star} \circ \Phi\right.$ is differentiable at $x$ for all $\left.n \geq 1\right\}$ has full measure in $X$, the mapping $G \circ \Phi: X \rightarrow V$ is differentiable almost everywhere.

Now, we can show the main theorem of this section, which gives a sufficient condition for the set of differentiable almost everywhere and Lipschitz vector-valued functions to approximate set of Lipschitz vectorvalued functions.

Theorem 3.7. Let $(X, d, \mu)$ be a metric measure space with a MDS where $(X, d)$ is doubling, and let $V$ be a Banach space. Then, for every Lipschitz mapping $f: X \rightarrow V$ and every $\varepsilon>0$, there is a differentiable almost everywhere and Lipschitz mapping $g: X \rightarrow V$ such that

$$
\|f(x)-g(x)\|<\varepsilon \quad \text { for all } x \in X \text {, and } \quad \operatorname{Lip}(g) \leq 2 C \lambda(X)^{2} \operatorname{Lip}(f),
$$

where $C$ is the universal constant given by Theorem 3.4.

Proof. Notice that since $(X, d)$ is doubling then it is separable. Now, recall that Ahanori proved in [1] that for any $\rho>0$, every separable metric space $(X, d)$ is $(6+\rho)$-Lipschitz isomorphic to a subset of the Banach space $c_{0}$. Thus, for any $\rho>0$, there is a mapping $\Phi: X \rightarrow c_{0}$ such that

$$
d(x, y) \leq\|\Phi(x)-\Phi(y)\| \leq(6+\rho) d(x, y) \quad \text { for all } x, y \in X .
$$

Later Assouad in [2] and Pelant in [26] refined this result by showing that every separable metric space 3embeds into $c_{0}$. Finally, Kalton and Lancien [21] constructed a 2-embedding (resp. 1-embedding) into $c_{0}$ for every separable (resp. proper) metric space.

Thus, since our metric space $(X, d)$ is separable, there is a mapping $\Phi: X \rightarrow c_{0}$ such that

$$
d(x, y) \leq\|\Phi(x)-\Phi(y)\| \leq 2 d(x, y) \quad \text { for all } x, y \in X .
$$

We claim that $\Phi(X)$ is doubling in $c_{0}$ with constant $\lambda(X)^{2}$. Indeed, for any $x \in X$ and $r>0$ we have that

$$
\Phi^{-1}(B(\Phi(x), r)) \subset B(x, r) \subset \bigcup_{j=1}^{\lambda(X)^{2}} B\left(x_{j}, \frac{r}{2^{2}}\right)
$$


since $\Phi^{-1}$ is 1-Lipschitz and $X$ is doubling. Now, using the fact that $\Phi$ is 2-Lipschitz,

$$
\begin{aligned}
B(\Phi(x), r) \cap \Phi(X) & \subset \bigcup_{j=1}^{\lambda(X)^{2}} \Phi\left(B\left(x_{j}, \frac{r}{2^{2}}\right)\right) \subset \bigcup_{j=1}^{\lambda(X)^{2}} B\left(\Phi\left(x_{j}\right), 2 \frac{r}{2^{2}}\right) \cap \Phi(X) \\
& \subset \bigcup_{j=1}^{\lambda(X)^{2}} B\left(\Phi\left(x_{j}\right), \frac{r}{2}\right) \cap \Phi(X),
\end{aligned}
$$

which proves the claim.

Moreover, if $f: X \rightarrow V$ is a Lipschitz map, then $f \circ \Phi^{-1}: \Phi(X) \rightarrow V$ is a $\operatorname{Lip}(f)$-Lipschitz map, and by Theorem 3.4 there is a Lipschitz extension $F: c_{0} \rightarrow V$ with $\operatorname{Lip}(F) \leq C \lambda(X)^{2} \operatorname{Lip}(f)$.

Given $\varepsilon>0$, adapting the proof of [15, Theorem 1] (see also [17, Theorem 74, pg 437]) we find a $C^{\infty}$ smooth, LFC- $\left\{e_{n}^{\star}\right\}_{n=1}^{\infty}$ and Lipschitz mapping $G: c_{0} \rightarrow V$ such that

$$
\|F(x)-G(x)\|<\varepsilon \quad \text { for all } x \in c_{0}, \text { and } \quad \operatorname{Lip}(G) \leq \operatorname{Lip}(F) .
$$

Let us take $g: X \rightarrow V$ defined as $g(x)=G \circ \Phi(x)$. Then

- $\operatorname{Lip}(g) \leq 2 C \lambda(X)^{2} \operatorname{Lip}(f)$,

- $\|f(x)-g(x)\|=\left\|\left(f \circ \Phi^{-1}\right) \circ \Phi(x)-G \circ \Phi(x)\right\|<\varepsilon$ for all $x \in X$, and

- by Lemma 3.6, $g$ is differentiable almost everywhere on $X$.

In the next proposition we show that subsets of doubling metric spaces are doubling too. We include it in this work as we lack a reference for it.

Proposition 3.8. Let $(X, d)$ be a doubling metric space and $A \subseteq X$. Then $(A, d)$ is doubling too with doubling constant at most $\lambda(X)^{2}$, where $\lambda(X)$ stands for the doubling constant of $X$.

Proof. Let $A \subseteq X$ and let $B_{A}(a, r)$ be a ball in $(A, d)$. We need to show that it can be covered by at most a given number $\lambda(A)$ of ball in $(A, d)$ with radius at most $r / 2$. Since $(X, d)$ is doubling and $B_{A}(a, r) \subseteq B_{X}(a, r)$, there are at most $\lambda(X)$ balls in $X$ such that

$$
B_{A}(a, r) \subseteq \bigcup_{i=1}^{\lambda(X)} B_{X}\left(x_{i}, r / 2\right) .
$$

Applying the doubling property again, each ball $B_{X}\left(x_{i}, r / 2\right)$ can be covered by, at most, $\lambda(X)$ balls with center in $X$ and radius $r / 4$. Therefore we have, at most, $\lambda(X)^{2}$ balls of radius $r / 4$ which union contains $B_{A}(a, r)$. Taking, for each of these balls, a point in the intersection of $A$ with it (when such intersection is nonempty) we obtain, at most, $\lambda(X)^{2}$ points in $A$ such that the union of balls with center in these points and radius $r / 2$ covers $B_{A}(a, r)$.

Corollary 3.9. Let $(X, d, \mu)$ be a metric measure space with a MDS where $(X, d)$ is doubling, and let $V$ be a Banach space. Then, for every subset $A \subset X$, every Lipschitz mapping $f: A \rightarrow V$ and every $\varepsilon>0$, there is a differentiable almost everywhere and Lipschitz mapping $g: X \rightarrow V$ such that

$$
\|f(x)-g(x)\|<\varepsilon \quad \text { for all } x \in A \text {, and } \quad \operatorname{Lip}(g) \leq 2 C \lambda(X)^{4} \operatorname{Lip}(f),
$$

where $C$ is the universal constant given by Theorem 3.4 .

Finally, notice that a doubling metric space is proper (i.e., closed and bounded subsets are compact) if and only if it is complete. Then, using the 1-embedding into $c_{0}$ for proper metric spaces given in [21], we obtain the last result of this section. 
Corollary 3.10. Let $(X, d, \mu)$ be a metric measure space with a MDS where $(X, d)$ is complete and doubling, and let $V$ be a Banach space. Then, for every Lipschitz mapping $f: X \rightarrow V$ and every $\varepsilon>0$, there is a differentiable almost everywhere and Lipschitz mapping $g: X \rightarrow V$ such that

$$
\|f(x)-g(x)\|<\varepsilon \quad \text { for all } x \in X \text {, and } \quad \operatorname{Lip}(g) \leq C \lambda(X) \operatorname{Lip}(f),
$$

where $C$ is the universal constant given by Theorem 3.4.

\section{Remark 3.11.}

(i) Let $\mathscr{C} \subset \operatorname{LIP}(X)$ such that $(X, d, \mu)$ has a $\mathscr{C}$-MDS, the main theorem holds whenever the functions $x \mapsto$ $d\left(x, x_{0}\right)$ belong to $\mathscr{C}$ for all $x_{0} \in X$. Indeed, following the proof of [27, Proposition 3.1.3, Proposition 3.1.2] (see also [16]) the Lipschitz embedding $\Phi$ into $c_{0}$ can be chosen with differentiable almost everywhere coordinate functions and Lipschitz constant less or equal to $4+r$ for any $r>0$.

(ii) Furthermore, the approximation mapping is differentiable everywhere on $X$ whenever the functions $x \mapsto$ $d\left(x, x_{0}\right)$ are differentiable everywhere but $x_{0}$.

\section{Extension of differentiable functions}

In this section we study the problem of extending differentiable functions from a metric space with a MDS as differentiable functions. We begin by obtaining some Lip-derivation inequalities for vector-valued functions similar to the one obtained in $[6,14]$ in the real-valued case.

Suppose that $(X, d, \mu)$ is a metric measure space with a MDS and $V$ is a Banach space. Given a mapping $f: X \rightarrow V$, the point-wise Lipschitz constant, $\operatorname{Lip}_{x}(f)$, at $x \in X$ is given by:

$$
\operatorname{Lip}_{x}(f)=\limsup _{y \rightarrow x} \frac{\|f(y)-f(x)\|}{d(y, x)} .
$$

A mapping $f: X \rightarrow V$ satisfying equation (2.1) on a chart $\left(X_{\alpha}, \mathbf{y}^{\alpha}\right)$ satisfies

$$
\operatorname{Lip}_{x}(f)=\operatorname{Lip}_{x}\left(\left\{\frac{d f}{d y_{m}^{\alpha}}(x)\right\}_{m=1}^{k(\alpha)} \cdot \mathbf{y}^{\alpha}\right) \leq \operatorname{Lip}\left(\mathbf{y}^{\alpha}\right) \sum_{m=1}^{k(\alpha)}\left\|\frac{d f}{d y_{m}^{\alpha}}(x)\right\|,
$$

for almost every $x \in X_{\alpha}$. The opposite inequality also holds, but a finer atlas must be chosen. Firstly, let us recall the real-valued case (see [6, Lemma 4.32] or [14, Lemma 5.1]).

Proposition 4.1. Let $(X, d, \mu)$ be a metric measure space with a MDS. Then, there is an atlas $\left\{\left(X^{\alpha}, \mathbf{y}^{\alpha}\right)\right\}_{\alpha \in \mathscr{A}}$ on $X$ such that for each $\alpha \in \mathscr{A}$ there exists a constant $C_{\alpha}>0$, which depends on the chart $\left(X_{\alpha}, \mathbf{y}^{\alpha}\right)$, such that for every differentiable almost everywhere function $f: X \rightarrow \mathbb{R}$

$$
\left\|d^{\alpha} f(x)\right\|_{\infty} \leq C_{\alpha} \operatorname{Lip}_{x}(f),
$$

for a.e. $x \in X_{\alpha}$.

Notice that $d f^{\alpha}(x)=\left\{\frac{d f}{d y_{m}^{\alpha}}(x)\right\}_{m=1}^{k(\alpha)} \in \mathbb{R}^{k(\alpha)}$ and $\left\|d f^{\alpha}(x)\right\|_{\infty}=\max _{1 \leq m \leq k(\alpha)}\left|\frac{d f}{d y_{m}^{\alpha}}(x)\right|$.

Proposition 4.2. Let $(X, d, \mu)$ be a metric measure space with a MDS and let $V$ be a Banach space. Then, for every differentiable almost everywhere mapping $f: X \rightarrow V$, we have that

$$
\max _{1 \leq m \leq k(\alpha)}\left\|\frac{d f}{d y_{m}^{\alpha}}(x)\right\| \leq C_{\alpha} \operatorname{Lip}_{x}(f),
$$

for a.e. $x \in X_{\alpha}$, where $\left\{\left(X^{\alpha}, \mathbf{y}^{\alpha}\right)\right\}_{\alpha \in \mathscr{A}}$ and $C_{\alpha}$ are the atlas on $X$ and the constants given in Proposition 4.1. 
Proof. Since $f: X \rightarrow V$ is differentiable almost everywhere, the function $\phi \circ f: X \rightarrow \mathbb{R}$ is differentiable almost everywhere for any $\phi \in V^{\star}$ (actually, they are differentiable at the points where $f$ is differentiable). Moreover, it is easy to see that

$$
d^{\alpha}(\phi \circ f)(x)=\left\{\frac{d(\phi \circ f)}{d y_{m}^{\alpha}}(x)\right\}_{m=1}^{k(\alpha)}=\left\{\phi\left(\frac{d f}{d y_{m}^{\alpha}}(x)\right)\right\}_{m=1}^{k(\alpha)} .
$$

Using Proposition 4.1

for a.e. $x \in X_{\alpha}$.

$$
\max _{1 \leq m \leq k(\alpha)}\left|\phi\left(\frac{d f}{d y_{m}^{\alpha}}(x)\right)\right| \leq C_{\alpha} \operatorname{Lip}_{x}(\phi \circ f) \leq C_{\alpha}\|\phi\| \operatorname{Lip}_{x}(f),
$$

Notice that the above inequality is satisfied for every $\phi \in V^{\star}$ and any point $x \in X_{\alpha}$ where $f$ is differentiable. Thus, for any $1 \leq m \leq k(\alpha)$ and any point $x \in X_{\alpha}$ where $f$ is differentiable, we choose $\phi \in V^{\star}$ such that $\|\phi\|=1$ and $\phi\left(\frac{d f}{d y_{m}^{\alpha}}(x)\right)=\left\|\frac{d f}{d y_{m}^{\alpha}}(x)\right\|$, and we obtain that

$$
\left\|\frac{d f}{d y_{m}^{\alpha}}(x)\right\|=\left|\phi\left(\frac{d f}{d y_{m}^{\alpha}}(x)\right)\right| \leq \max _{1 \leq m \leq k(\alpha)}\left|\phi\left(\frac{d f}{d y_{m}^{\alpha}}(x)\right)\right| \leq C_{\alpha} \operatorname{Lip}_{x}(f) .
$$

To sum up, inequality (4.1) holds for any $x \in X_{\alpha}$ where $f$ is differentiable.

To show the main result of this section, we need the following lemma.

Lemma 4.3. Let $(X, d, \mu)$ be a metric measure space with a MDS where $(X, d)$ is doubling, let $V$ be a Banach space and $A \subset X$. Then, for every continuous mapping $F: X \rightarrow V$ such that $F_{\left.\right|_{A}}$ is Lipschitz, and every $\varepsilon>0$, there exists a differentiable almost everywhere mapping $G: X \rightarrow V$ such that:

(i) $\|F(x)-G(x)\|<\varepsilon$ for all $x \in X$,

(ii) $\operatorname{Lip}\left(G_{\left.\right|_{A}}\right) \leq 2 C \lambda(X)^{4} \operatorname{Lip}\left(F_{\left.\right|_{A}}\right)$, where $C$ is the universal constant given in Theorem 3.4.

(iii) In addition, if $F$ is Lipschitz, then there exists a constant $C_{1} \geq 2 C \lambda(X)^{4}$, depending only on the doubling constant of $X$, such that the mapping $G$ can be chosen to be Lipschitz on $X$ and $\operatorname{Lip}(G) \leq C_{1} \operatorname{Lip}(F)$.

Proof. Assume that the mapping $F: X \rightarrow V$ is continuous on $X$ and $F_{\left.\right|_{A}}$ is Lipschitz. By Theorem 3.1 there is a differentiable almost everywhere mapping $h: X \rightarrow V$, such that $\|F(x)-h(x)\|<\varepsilon$ for all $x \in X$. Let us apply Corollary 3.9 to $F_{\left.\right|_{A}}$ to obtain a differentiable almost everywhere and Lipschitz mapping $g: X \rightarrow V$ such that

(a) $\|F(x)-g(x)\|<\varepsilon / 4$ for all $x \in A$, and

(b) $\operatorname{Lip}(g) \leq 2 C \lambda(X)^{4} \operatorname{Lip}\left(F_{\left.\right|_{A}}\right)$.

Consider the open sets $D=\{x \in X:\|F(x)-g(x)\|<\varepsilon / 2\}$ and the closed set $C=\{x \in X:\|F(x)-g(x)\| \leq \varepsilon / 4\}$ in $X$. Then $A \subset C \subset D$. There is a differentiable almost everywhere function $u: X \rightarrow[0,1]$ such that

$$
u(x)= \begin{cases}1 & \text { if } x \in C \\ 0 & \text { if } x \in X \backslash D .\end{cases}
$$

Indeed, since $C \cap(X \backslash D)=\emptyset$, the function

$$
p(x)=\frac{\operatorname{dist}(x, X \backslash D)}{\operatorname{dist}(x, C)+\operatorname{dist}(x, X \backslash D)}
$$

is continuous on $X, p(C)=1$ and $p(X \backslash D)=0$. Using Theorem 3.1, there is a differentiable almost everywhere function $q: X \rightarrow \mathbb{R}$ such that $|p(x)-q(x)|<\frac{1}{4}$. Let us take a $C^{1}$ smooth function $\theta: \mathbb{R} \rightarrow[0,1]$ such that $\theta(t)=0$ whenever $t \leq 1 / 4$ and $\theta(t)=1$ whenever $t \geq 3 / 4$. Then, $u(x)=\theta(q(x))$ satisfies the desired properties.

Let us define $G: X \rightarrow V$ as

$$
G(x):=u(x) g(x)+(1-u(x)) h(x) .
$$

It is clear that $G$ is a differentiable almost everywhere mapping. Since $u(x)=0$ for all $x \in X \backslash D$, we deduce that

$$
\|F(x)-G(x)\|=\|F(x)-h(x)\|<\varepsilon \quad \text { for all } x \in X \backslash D .
$$


Now, if $x \in D$, then

$$
\|F(x)-G(x)\| \leq u(x)|| F(x)-g(x)\|+(1-u(x))|| F(x)-h(x)\| \leq u(x) \varepsilon / 2+(1-u(x)) \varepsilon \leq \varepsilon .
$$

Finally, since $u(x)=1$ and $G(x)=g(x)$ for every $x \in C$, we obtain that $\operatorname{Lip}\left(G_{\left.\right|_{A}}\right)=\operatorname{Lip}\left(g_{\left.\right|_{A}}\right) \leq 2 C \lambda(X)^{4} \operatorname{Lip}\left(F_{\left.\right|_{A}}\right)$.

To prove the last part, let us now assume that $F$ is Lipschitz on the whole $X$. Let us apply Theorem 3.7 and Corollary 3.9 to $F$ and $F_{\left.\right|_{A}}$ to obtain differentiable almost everywhere mappings $g$ and $h$ from $X$ into $V$ such that

(a) $\|F(x)-g(x)\|<\varepsilon / 4$ for all $x \in A$,

(b) $\|F(x)-h(x)\|<\varepsilon$ for all $x \in X$,

(c) $\operatorname{Lip}(g) \leq 2 C \lambda(X)^{4} \operatorname{Lip}\left(F_{\left.\right|_{A}}\right)$ and $\operatorname{Lip}(h) \leq 2 C \lambda(X)^{2} \operatorname{Lip}(F)$.

We take again the open subsets $B, D$ and the closed subset $C$ as earlier in this proof. Notice that

$$
\operatorname{dist}(C, X \backslash D) \geq \frac{\varepsilon}{4\left(\operatorname{Lip}(F)+2 C \lambda(X)^{4} \operatorname{Lip}\left(F_{\left.\right|_{A}}\right)\right)}=\varepsilon^{\prime} .
$$

We claim that there is a differentiable almost everywhere and Lipschitz function $u: X \rightarrow[0,1]$ such that

$$
u(x)=\left\{\begin{array}{ll}
1 & \text { if } x \in C \\
0 & \text { if } x \in X \backslash D
\end{array} \quad \text { and } \quad \operatorname{Lip}(u) \leq \frac{4\left(\operatorname{Lip}(F)+2 C \lambda(X)^{4} \operatorname{Lip}\left(F_{\left.\right|_{A}}\right)\right)}{\varepsilon} .\right.
$$

In fact, let us take a Lipschitz function $\theta: \mathbb{R} \rightarrow[0,1]$ such that $\theta(t)=0$ whenever $t \leq 0, \theta(t)=1$ whenever $t \geq \varepsilon^{\prime}$ and $\operatorname{Lip}(\theta)=1 / \varepsilon^{\prime}$. Thus, the function $u(x)=\theta(\operatorname{dist}(x, X \backslash D))$ is Lipschitz, so it is differentiable almost everywhere, $u(C)=1, u(X \backslash D)=0$ and $\operatorname{Lip}(\theta) \leq 1 / \varepsilon^{\prime}$.

Let us now consider $G: X \rightarrow V$ as

$$
G(x)=u(x) g(x)+(1-u(x)) h(x) .
$$

Clearly $G$ is differentiable almost everywhere on $X$. We follow the above proof to obtain that

(i) $\|F(x)-G(x)\|<\varepsilon$ on $X$,

(ii) $\operatorname{Lip}\left(G_{\left.\right|_{A}}\right)=\operatorname{Lip}\left(g_{\left.\right|_{A}}\right) \leq 2 C \lambda(X)^{4} \operatorname{Lip}\left(F_{\left.\right|_{A}}\right)$.

Additionally, if $y, z \in X \backslash D$, then $u(y)=0, u(z)=0, G(y)=h(y), G(z)=h(z)$, and $\|G(y)-G(z)\|=\| h(y)-$ $h(z) \| \leq 2 C \lambda(X)^{2} \operatorname{Lip}(F) d(y, z)$. For $y, z \in \bar{D}$, we have

$$
\begin{aligned}
\| G(y) & -G(z)\|=\| g(y) u(y)+h(y)(1-u(y))-g(z) u(z)-h(z)(1-u(z)) \| \\
& \leq\|g(y)(u(y)-u(z))+h(y)(u(z)-u(y))\|+\|u(y)(g(y)-g(z))+(1-u(y))(h(y)-h(z))\| \\
& \leq\|(g(y)-F(y))(u(y)-u(z))+(h(y)-F(y))(u(z)-u(y))\|+2 C \lambda(X)^{4} \operatorname{Lip}(F) d(y, z) \\
& \leq\left((\varepsilon / 2+\varepsilon) \operatorname{Lip}(u)+4 C \lambda(X)^{4} \operatorname{Lip}(F)\right) d(y, z) \\
& \leq\left(6+16 C \lambda(X)^{4}\right) \operatorname{Lip}(F) d(y, z) .
\end{aligned}
$$

The case $z \in X \backslash D$ and $y \in D$ follows the same way as the previous one. We define $C_{1}:=6+16 C \lambda(X)^{4}$ and, finally, obtain that $\operatorname{Lip}(G) \leq C_{1} \operatorname{Lip}(F)$.

Equation (2.1) can be also written as

$$
f(z)-f(x)-\sum_{m=1}^{k(\alpha)} \frac{d f}{d y_{m}^{\alpha}}(x)\left(y_{m}^{\alpha}(z)-y_{m}^{\alpha}(x)\right)=o(d(z, x))
$$

for a.e. $x \in X_{\alpha}$. This expression inspires the mean value condition we will need to impose in order to obtain our results. This mean value condition was proved to be needed for $C^{1}$ extension of vector valued mappings in [20] when working this problem in the context of Banach spaces. We adapt the notion given in [20] to our context in the following definition. 
Definition 4.4. Let $(X, d, \mu)$ be a metric measure space with a MDS, consider $\left\{\left(X_{\alpha}, \mathbf{y}^{\alpha}\right)\right\}_{\alpha \in \mathscr{A}}$ an atlas on it. Let $V$ be a Banach space and $A \subset X$ a measurable subset with $\mu(A)>0$. We say that a differentiable almost everywhere mapping $f: A \rightarrow V$ satisfies the mean value condition on $A$ if for every $\alpha$, almost every $x \in A \cap X_{\alpha}$ and every $\varepsilon>0$, there is an open ball $B(x, r)$ in $X$ such that

$$
\left\|f(z)-f(y)-\sum_{m=1}^{k(\alpha)} \frac{d f}{d y_{m}^{\alpha}}(x)\left(y_{m}^{\alpha}(z)-y_{m}^{\alpha}(y)\right)\right\| \leq \varepsilon d(z, y),
$$

for every $z, y \in A \cap X_{\alpha} \cap B(x, r)$.

The following theorem is the main result in this section. It is rather an approximation result than an extension one as it shows that continuous extensions can be approached by smooth mappings in a precise way that can be useful to obtain the extensions result we are seeking. How to apply this result will be show later.

Theorem 4.5. Let $(X, d, \mu)$ be a metric measure space with a MDS where $(X, d)$ is doubling, let $V$ be a Banach space, $A \subset X$ a closed subset and $f: A \rightarrow V$ a mapping satisfying the mean value condition. Let us consider $F: X \rightarrow V$ a continuous extension of $f$ to $X$. Then, for every $\varepsilon>0$ there exists a differentiable almost everywhere $G: X \rightarrow V$ such that

(i) $\|F(x)-G(x)\|<\varepsilon$ on $X$, and

(ii) $\operatorname{Lip}\left(f-G_{\left.\right|_{A}}\right)<\varepsilon$.

(iii) Furthermore, assume that $f$ is Lipschitz on $A$ and $F$ is a Lipschitz extension of $f$ to $X$. Then the function $G$ can be chosen to be Lipschitz on $X$ and $\operatorname{Lip}(G) \leq C \operatorname{Lip}(F)$ for a certain constant $C$.

Proof. Assume that $F$ is a continuous extension of $f$. Since $X$ is a separable metric space, $A \subset X$ is a closed subspace and $f$ satisfies the mean value condition on $A$, there exists $\left\{B\left(x_{n}, r_{n}\right)\right\}_{n \in \mathbb{N}}$ a covering of $A$ by open balls of $X$, with centers $x_{n} \in A$ such that

$$
\left\|f(z)-f(y)-\sum_{m=1}^{k(\alpha)} \frac{d f}{d y_{m}^{\alpha}}\left(x_{n}\right)\left(y_{m}^{\alpha}(z)-y_{m}^{\alpha}(y)\right)\right\| \leq \frac{\varepsilon}{8 C_{0}} d(z, y),
$$

for every $z, y \in A \cap X_{\alpha} \cap B\left(x_{n}, r_{n}\right)$, where $C_{0}=2 C \lambda(X)^{4}$.

Let us define $T_{n}$ as the first order Taylor Polynomial of $f$ at $x_{n}$ given by

$$
T_{n}(x)=f\left(x_{n}\right)+\sum_{m=1}^{k(\alpha)} \frac{d f}{d y_{m}^{\alpha}}\left(x_{n}\right)\left(y_{m}^{\alpha}(x)-y_{m}^{\alpha}\left(x_{n}\right)\right),
$$

for $x \in X$. Notice that $T_{n}$ satisfies the following properties:

(1) $T_{n}$ is differentiable almost everywhere on $X$,

(2) $\frac{d T_{n}}{d y_{m}^{\alpha}}(x)=\frac{d f}{d y_{m}^{\alpha}}\left(x_{n}\right)$ for almost every $x$ in $X$ and $1 \leq m \leq k(\alpha)$, and

(3) for all $z, y \in A \cap X_{\alpha} \cap B\left(x_{n}, r_{n}\right)$,

$$
\begin{gathered}
\left\|\left(T_{n}-F\right)(y)-\left(T_{n}-F\right)(z)\right\|= \\
=\left\|f(z)-f(y)-\sum_{m=1}^{k(\alpha)} \frac{d f}{d y_{m}^{\alpha}}\left(x_{n}\right)\left(y_{m}^{\alpha}(z)-y_{m}^{\alpha}(y)\right)\right\| \leq \frac{\varepsilon}{8 C_{0}} d(z, y) .
\end{gathered}
$$

Thus, $\operatorname{Lip}\left(\left.\left(T_{n}-F\right)\right|_{A \cap X_{\alpha} \cap B\left(x_{n}, r_{n}\right)}\right) \leq \frac{\varepsilon}{8 C_{0}}$.

By Theorem 3.1 there is a differentiable almost everywhere mapping $F_{0}: X \rightarrow V$ such that $\left\|F(x)-F_{0}(x)\right\|<$ $\frac{\varepsilon}{2}$ for every $x \in X$.

Let us denote the open set $B_{0}:=X \backslash A$. Then, the collection $\left\{B\left(x_{n}, r_{n}\right)\right\}_{n=1}^{\infty} \cup B_{0}$ is a covering of $X$ by open sets. In the same way as the proof of Theorem 3.1, there is a partition of unity $\left\{\varphi_{n}\right\}_{n=0}^{\infty}$ of differentiable almost everywhere and Lipschitz functions such that 
- for each $x \in X$ there is a neighborhood of $x$ where all but a finite number of the functions are 0 ,

- $\operatorname{supp} \varphi_{n}=\left\{x \in X: \varphi_{n}(x) \neq 0\right\} \subset B\left(x_{n}, r_{n}\right)$, supp $\varphi_{0} \subset B_{0}$, and

- $\sum_{n=0}^{\infty} \varphi_{n}=1$.

Let us define $L_{n}:=\max \left\{\operatorname{Lip}\left(\varphi_{n}\right), 1\right\}$ for every $n \geq 0$. Now, for every $n \in \mathbb{N}$ we apply Lemma 4.3 to $T_{n}-F$ on $A \cap X_{\alpha} \cap B\left(x_{n}, r_{n}\right)$ to obtain a differentiable almost everywhere mapping $\delta_{n}: X \longrightarrow V$ so that

$$
\left\|T_{n}(x)-F(x)-\delta_{n}(x)\right\|<\frac{\varepsilon}{2^{n+2} L_{n}} \text { for every } x \in X
$$

and

$$
\operatorname{Lip}\left(\delta_{\left.n\right|_{A \cap X_{\alpha} \cap B\left(x_{n}, r_{n}\right)}}\right) \leq \frac{\varepsilon}{8} .
$$

Let us define

$$
G(x)=\varphi_{0}(x) F_{0}(x)+\sum_{n=1}^{\infty} \varphi_{n}(x)\left(T_{n}-\delta_{n}\right)(x) .
$$

The mapping $G$ is differentiable almost everywhere since $\left\{\varphi_{n}\right\}_{n=0}^{\infty}$ is locally finitely nonzero. For every $x \in X$

$$
\begin{gathered}
\|G(x)-F(x)\| \leq \varphi_{0}(x)\left\|F_{0}(x)-F(x)\right\|+\sum_{n \geq 1} \varphi_{n}(x)\left\|T_{n}(x)-\delta_{n}(x)-F(x)\right\| \leq \\
\leq \sum_{n \geq 0} \varphi_{n}(x) \frac{\varepsilon}{2}<\varepsilon .
\end{gathered}
$$

Let us prove that $\operatorname{Lip}\left(f-G_{\left.\right|_{A}}\right)<\varepsilon$. In order to simplify the notation let us write $F_{z}:=\left\{n \geq 0: \varphi_{n}(z) \neq 0\right\}$ for $z \in X$, and $S_{n}(y):=T_{n}(y)-\delta_{n}(y)-f(y)$ for $y \in A$. Now, we obtain

$$
\begin{aligned}
& \begin{array}{l}
\|(G(y)-f(y))-(G(z)-f(z))\|= \\
=\left\|\sum_{n \in F_{y}} \varphi_{n}(y) S_{n}(y)-\sum_{n \in F_{z}} \varphi_{n}(z) S_{n}(z)-\sum_{n \in F_{y}} \varphi_{n}(z) S_{n}(y)+\sum_{n \in F_{y}} \varphi_{n}(z) S_{n}(y)\right\| \\
=\|\left(\sum_{n \in F_{y}} \varphi_{n}(y) S_{n}(y)-\sum_{n \in F_{y}} \varphi_{n}(z) S_{n}(y)\right)+\left(\sum_{n \in F_{y}} \varphi_{n}(z) S_{n}(y)-\sum_{n \in F_{z} \cap F_{y}} \varphi_{n}(z) S_{n}(z)\right) \\
\quad+\left(\sum_{n \in F_{z} \backslash F_{y}} \varphi_{n}(y) S_{n}(z)-\sum_{n \in F_{z} \backslash F_{y}} \varphi_{n}(z) S_{n}(z)\right) \| \\
\leq \sum_{n \in F_{y}}\left|\varphi_{n}(y)-\varphi_{n}(z)\right|\left\|S_{n}(y)\right\|+\sum_{n \in F_{z} \cap F_{y}} \varphi_{n}(z)\left\|S_{n}(y)-S_{n}(z)\right\| \\
\quad+\sum_{n \in F_{z} \backslash F_{y}}\left|\varphi_{n}(y)-\varphi_{n}(z)\right|\left\|S_{n}(z)\right\| \leq \sum_{n \in F_{y}} L_{n} d(y, z) \frac{\varepsilon}{2^{n+2} L_{n}} \\
\quad+\sum_{n \in F_{z} \cap F_{y}} \varphi_{n}(z) \frac{\varepsilon}{4} d(y, z)+\sum_{n \in F_{z} \backslash F_{y}} L_{n} d(y, z) \frac{\varepsilon}{2^{n+2} L_{n}}<\varepsilon d(y, z) .
\end{array}
\end{aligned}
$$

Let us now consider the case when $F$ is a Lipschitz extension of $f$ on $X$. In this case, we can assume that $f$ is not constant (otherwise the assertion is trivial) and thus $\operatorname{Lip}(F) \geq \operatorname{Lip}(f)>0$. Let us fix $0<\varepsilon<\operatorname{Lip}(F)$. If we follow the above construction for the open covering $\left\{B\left(x_{n}, r_{n}\right)\right\}_{n=1}^{\infty} \cup B_{0}$ of $X$, we additionally obtain

(4) $T_{n}-F$ is Lipschitz on $X$ and, by Proposition 4.2,

$$
\operatorname{Lip}\left(T_{n}\right) \leq \operatorname{Lip}\left(\mathbf{y}^{\alpha}\right) \sum_{m=1}^{k(\alpha)}\left\|\frac{d f}{d y_{m}^{\alpha}}\left(x_{n}\right)\right\| \leq \operatorname{Lip}\left(\mathbf{y}^{\alpha}\right) k(\alpha) C_{\alpha} \operatorname{Lip}(f) \leq K_{\alpha} \operatorname{Lip}(f),
$$

where $K_{\alpha}=k(\alpha) C_{\alpha} \operatorname{Lip}\left(\mathbf{y}^{\alpha}\right)$. Thus, $\operatorname{Lip}\left(T_{n}-F\right) \leq K_{\alpha} \operatorname{Lip}(f)+\operatorname{Lip}(F)$ for every $n \in \mathbb{N}$.

Also, the construction of the Lipschitz partition of unity $\left\{\varphi_{n}\right\}_{n \geq 0}$ and the definition of $L_{n}$ are similar to the previous case. 
Now, for any $n \in \mathbb{N}$, we apply Lemma 4.3 to $T_{n}-F$ on $A \cap X_{\alpha} \cap B\left(x_{n}, r_{n}\right)$ to obtain a differentiable almost everywhere mapping $\delta_{n}: X \rightarrow V$ satisfying the properties of the previous case and

$$
\operatorname{Lip}\left(\delta_{n}\right) \leq C_{1} \operatorname{Lip}\left(T_{n}-F\right) \leq C_{1}\left(K_{\alpha} \operatorname{Lip}(f)+\operatorname{Lip}(F)\right) .
$$

Besides, by applying Corollary 3.9, we select a differentiable almost everywhere mapping $F_{0}: X \rightarrow V$, such that

$$
\left\|F_{0}(x)-F(x)\right\|<\frac{\varepsilon}{2^{2} L_{0}} \text { for every } x \in X \text { and } \operatorname{Lip}\left(F_{0}\right) \leq 2 C \lambda(X)^{4} \operatorname{Lip}(F) .
$$

Similarly to the first case, the definition of $G$ is

$$
G(x)=\varphi_{0}(x) F_{0}(x)+\sum_{n=1}^{\infty} \varphi_{n}(x)\left(T_{n}-\delta_{n}\right)(x) .
$$

The proofs that $G$ is differentiable almost everywhere, $\|G(x)-F(x)\|<\varepsilon$ for all $x \in X$ and $\operatorname{Lip}\left(f-G_{\left.\right|_{A}}\right)<\varepsilon$ follow along the same lines. To show that $G$ is Lipschitz, notice that, from the fact that the mappings $\left\{\varphi_{n}\right\}$ are a partition of unity,

$$
\begin{gathered}
G(z)-G(y)=\left(\varphi_{0}(z)-\varphi_{0}(y)\right)\left(F_{0}(z)-F(z)\right)+\varphi_{0}(y)\left(F_{0}(z)-F_{0}(y)\right)+ \\
+\sum_{n \geq 1}\left(\varphi_{n}(z)-\varphi_{n}(y)\right)\left(T_{n}(z)-\delta_{n}(z)-F(z)\right)+\sum_{n \geq 1} \varphi_{n}(y)\left(\left(T_{n}(z)-\delta_{n}(z)\right)-\left(T_{n}(y)-\delta_{n}(y)\right)\right) .
\end{gathered}
$$

Therefore

$$
\begin{aligned}
\|G(z)-G(y)\| \leq & \left\|\left(\varphi_{0}(z)-\varphi_{0}(y)\right)\left(F_{0}(z)-F(z)\right)\right\|+\left\|\varphi_{0}(y)\left(F_{0}(z)-F_{0}(y)\right)\right\| \\
& +\left\|\sum_{n \geq 1}\left(\varphi_{n}(z)-\varphi_{n}(y)\right)\left(T_{n}(z)-\delta_{n}(z)-F(z)\right)\right\|+\left\|\sum_{n \geq 1} \varphi_{n}(y)\left(\left(T_{n}(z)-\delta_{n}(z)\right)-\left(T_{n}(y)-\delta_{n}(y)\right)\right)\right\| \\
\leq & \frac{\varepsilon}{2^{2}} d(y, z)+2 C \lambda(X)^{4} \operatorname{Lip}(F) d(y, z)+\sum_{n=1}^{\infty} \frac{\varepsilon}{2^{n+2}} d(y, z)+\left(1+C_{1}\right)\left(K_{\alpha} \operatorname{Lip}(f)+\operatorname{Lip}(F)\right) d(y, z) \\
\leq & \left(\varepsilon+2 C \lambda(X)^{4} \operatorname{Lip}(F)+\left(1+C_{1}\right)\left(K_{\alpha} \operatorname{Lip}(f)+\operatorname{Lip}(F)\right)\right) d(y, z) .
\end{aligned}
$$

We close the paper by showing how this result can be applied to obtain smooth extensions of smooth mappings. We begin with a convergence theorem.

Theorem 4.6. Let $(X, d, \mu)$ be a metric measure space with a MDS and $V$ a Banach space. If $\left\{f_{n}\right\}$ is a sequence of differentiable almost everywhere functions from $X$ to $V$ and $f: X \rightarrow V$ is such that

(i) $\operatorname{Lip}_{x}\left(f_{n}-f\right) \rightarrow 0$ for almost every $x \in X$, and

(ii) there exists a function $g: X \rightarrow V$ such that $d f_{n}^{\alpha}(x) \rightarrow g(x)$ for almost every $x \in X$,

then, $f$ is differentiable for almost every $x \in X$ and $d f^{\alpha}(x)=g(x)$.

Proof. We have to show that $\operatorname{Lip}_{x}\left(f-g(x) y^{\alpha}\right)=0$ for almost every $x \in X$, that is, for $\varepsilon>0$ there exists $\delta>0$ such that

$$
\sup \left\{\frac{\left\|f(z)-f(x)-g(x)\left(y^{\alpha}(z)-y^{\alpha}(x)\right)\right\|}{d(z, x)}: z \in B(x, \delta) \backslash\{x\}\right\}<\varepsilon .
$$

Let us fix $x \in X$ so that (i) and (ii) hold. From (i), there exists $n_{0} \in \mathbb{N}$ such that for $n \geq n_{0}$ we have that $\operatorname{Lip}_{x}\left(f_{n}-f\right)<\varepsilon / 3$. Therefore, there exists $\delta_{1}>0$ such that

$$
\sup \left\{\frac{\left\|\left(f_{n}-f\right)(z)-\left(f_{n}-f\right)(x)\right\|}{d(z, x)}: z \in B\left(x, \delta_{1}\right) \backslash\{x\}\right\}<\varepsilon / 3 .
$$

From (ii) and the fact the mappings $y^{\alpha}$ are Lipschitz by definition, there exists $n_{1} \in \mathbb{N}$ such that for $n \geq n_{1}$ we have that

$$
\left\|d f_{n}^{\alpha}(x)-g(x)\right\|<\frac{\varepsilon}{3 \operatorname{Lip}\left(y^{\alpha}\right)} .
$$


Let us take now $N=\max \left\{n_{0}, n_{1}\right\}$, since $f_{N}$ is differentiable almost everywhere, there exists $\delta>0$, we can choose it so that $\delta<\delta_{1}$, such that

$$
\sup \left\{\frac{\left\|f_{N}(z)-f_{N}(x)-d f_{N}^{\alpha}(x)\left(y^{\alpha}(z)-y^{\alpha}(x)\right)\right\|}{d(z, x)}: z \in B(x, \delta) \backslash\{x\}\right\}<\varepsilon / 3 .
$$

Then, $z \in B(x, \delta) \backslash\{x\}$,

$$
\begin{array}{r}
\frac{\left\|f(z)-f(x)-g(x)\left(y^{\alpha}(z)-y^{\alpha}(x)\right)\right\|}{d(z, x)} \leq \frac{\left\|f_{N}(z)-f_{N}(x)-d f_{N}^{\alpha}(x)\left(y^{\alpha}(z)-y^{\alpha}(x)\right)\right\|}{d(z, x)} \\
+\frac{\left\|\left(f_{N}-f\right)(z)-\left(f_{N}-f\right)(x)\right\|}{d(z, x)}+\frac{\left\|d f_{N}^{\alpha}(x)-g(x)\right\| \cdot\left\|y^{\alpha}(z)-y^{\alpha}(x)\right\|}{d(z, x)}<\varepsilon,
\end{array}
$$

which completes the proof.

We obtain the following corollary.

Corollary 4.7. Under the conditions of the above theorem, given a sequence of differentiable almost everywhere functions $f_{n}: X \rightarrow V$ such that

(i) $\sum_{n=1}^{\infty} f_{n}(x)<\infty$ for $x \in X$, and

(ii) $\operatorname{Lip}_{x}\left(\sum_{n=N+1}^{\infty} f_{n}\right) \rightarrow 0$ as $N \rightarrow \infty$, for almost every $x \in X$,

then, $f(x)=\sum f_{n}(x)$ is differentiable almost everywhere in $X$.

Proof. We will apply Theorem 4.6 for functions $\sum_{j=1}^{n} f_{j}$. We need the function $g(x)=\sum_{n=1}^{\infty} d f_{n}^{\alpha}(x)$. This function is well defined due to Proposition 4.2 and (ii). Also, (ii) in Theorem 4.6 follows from Proposition 4.2 and (ii) in this corollary. Therefore, $f$ is differentiable almost everywhere.

We go next with the final result of this paper where we provide existence of smooth extensions. We will require of a very strong use of the mean valued condition. Of course, the mean valued condition as required in the next statement can be weakened by imposing it only to the mappings that are needed in the proof, however we impose it on all differentiable mappings so it makes the exposition easier.

Theorem 4.8. Let $(X, d, \mu)$ be a metric measure space with a MDS where $(X, d)$ is doubling, let $V$ be a Banach space and $A \subset X$ a closed subset with $\mu(A)>0$. Let us supposed that any differentiable almost everywhere mapping from $X$ to $V$ satisfies the mean value condition on $A$. Then, given a differentiable almost everywhere and Lipschitz mapping from $A$ to $V$, there is a differentiable almost everywhere mapping $F: X \rightarrow V$ such that $F_{\left.\right|_{A}}=f$.

Moreover, if the mapping $f$ is Lipschitz, then the differentiable almost everywhere extension $F: X \rightarrow V$ can be chosen to be Lipschitz with $\operatorname{Lip}(F) \leq C \operatorname{Lip}(f)$, where $C \geq 1$ is a constant that only depends on $X$.

Proof. By Theorem 4.5, there exists a differentiable almost everywhere and Lipschitz $g_{1}: X \rightarrow V$ such that

(i) $\left\|f(x)-g_{1}(x)\right\|<1 / 2$ en $A$.

(ii) $\operatorname{Lip}\left(f-g_{\left.1\right|_{A}}\right)<1 / 2$.

(iii) $\operatorname{Lip}\left(g_{1}\right) \leq C \operatorname{Lip}(f)$.

Let us take $f-g_{1_{A}}: A \rightarrow V$, since we are assuming all differentiable functions satisfy the mean value condition, we can apply Theorem 4.5, for globally defined Lipschitz functions on the whole $X$, again and so there exists a differentiable almost everywhere and Lipschitz $g_{2}: X \rightarrow V$ such that

(i) $\left\|f(x)-g_{1}(x)-g_{2}(x)\right\|<1 / 2^{2}$ en $A$.

(ii) $\operatorname{Lip}\left(f-\left(g_{1}+g_{2}\right)_{\left.\right|_{A}}\right)<1 / 2^{2}$.

(iii) $\operatorname{Lip}\left(g_{2}\right) \leq C \operatorname{Lip}\left(f-g_{\left.1\right|_{A}}\right) \leq C / 2$.

Proceeding in this way, for all $n \geq 2$ there exists a differentiable almost everywhere and Lipschitz mapping $g_{n}: X \rightarrow V$ such that 
(i) $\left\|f(x)-\sum_{j=1}^{n} g_{j}(x)\right\|<1 / 2^{n}$ in $A$.

(ii) $\operatorname{Lip}\left(f-\left(\sum_{j=1}^{n} g_{j}\right)_{\mid A}\right)<1 / 2^{n}$.

(iii) $\operatorname{Lip}\left(g_{n}\right) \leq C / 2^{n-1}$.

We define now $g(x)=\sum_{n=1}^{\infty} g_{n}(x)$ for $x \in X$. Mapping $g$ verifies:

(i) $g(x)=f(x)$ for $x \in A$.

(ii) $\operatorname{Lip}(g) \leq \sum_{n=1}^{\infty} \operatorname{Lip}\left(g_{n}\right) \leq \operatorname{Lip}\left(g_{1}\right)+\sum_{n=2}^{\infty} C / 2^{n-1} \leq C(\operatorname{Lip}(f)+1)$.

(iii) For $x \in X$, take $a \in A$, and so $\|g(x)\| \leq\|g(x)-g(a)\|+\|f(a)\| \leq \operatorname{Lip}(g) d(x, a)+\|f(a)\|<\infty$.

(iv) $\operatorname{Lip}\left(\sum_{n=N+1}^{\infty} g_{n}\right) \leq \sum_{n=N+1}^{\infty} \operatorname{Lip}\left(g_{n}\right) \leq C \sum_{n=N+1}^{\infty} 1 / 2^{n-1}=C / 2^{N-1} \rightarrow 0$.

Finally, from Corollary 4.7, $\mathrm{g}$ is differentiable almost everywhere and the theorem is proved.

Remark 4.9. As final remark we point out that the mean value condition was needed in the studies in Banach spaces because they were looking for $C^{1}$ extensions. Since being of class $C^{1}$ has not been considered at all in this work, the remaining question is not if the strong assumptions on the mean value condition may be weakened but rather if it can be completely dropped.

Aknowledgement: The research of the first author was supported by Ministerio de Ciencia, Innovación y Universidades del Gobierno de España through the grant PGC2018-098474-B-C21, Plan Estatal 2017-2020 Generación Conocimiento - Proyectos I+D+i.

Conflict of interest: Authors state no conflict of interest.

\section{References}

[1] I. Aharoni, Every separable metric space is Lipschitz equivalent to a subset of $c_{0}^{+}$, Israel J. Math 19 (1974), 284-291.

[2] P. Assouad, Remarques sur un article de Israel Aharoni sur les prolongements lipschitziens dans $c_{0}$ (Israel J. Math. 19 (1974), 284-291), Israel J. Math. 31 (1978), 97-100.

[3] D. Azagra, R. Fry and L. Keener, Smooth extension of functions on separable Banach spaces, Math. Ann. 347 (2) (2010), 285-297.

[4] D. Bate, Structure of measures in Lipschitz differentiability spaces, J. Amer. Math. Soc. 28 (2) (2015), 421-482.

[5] R. Bonic and J. Frampton, Smooth functions on Banach manifolds, J. Math. Mech. 28 (15) (1966), 877-898.

[6] J. Cheeger, Differentiability of Lipschitz functions on metric measure spaces, Geom. Funct. Anal. 9 (3) (1999), 428-517.

[7] M. Cepedello-Boiso and P. Hájek, Analytic approximations of uniformly continuous functions in real Banach spaces, J. Math. Anal. Appl. 256 (1) (2001), 80-98.

[8] M. Fabian and V. Zizler, Norms that locally depend on countable many linear functionals, Extracta Math. 16 (2) (2001), 259 278.

[9] C. Fefferman, A sharp form of Whitney's extension theorem, Ann. of Math. 161 (2) (2005), 509-577.

[10] C. Fefferman, Whitney's extension problem for $\mathscr{C}^{m}$, Ann. of Math. 164 (2) (2006), 313-359.

[11] R. Fry, Analytic approximation on $c_{0}$, J. Funct. Anal. 158 (2) (1998), 509-520.

[12] G. Glaeser, Ėtude de quelques algèbres tayloriennes, J. Analyse Math. 6 (1958), 1-124.

[13] J. Heinonen, Lectures on Analysis on Metric Spaces, Springer Verlag, 2001.

[14] J. Gong, Measurable differentiable structures on doubling metric spaces, arXiv:1110.4279v2.

[15] P. Hájek and M. Johanis, Uniformly Gâteaux smooth approximations on $c_{0}(\Gamma)$, J. Math. Anal. Appl. 350 (2) (2009), 623-629.

[16] P. Hájek and M. Johanis, Smooth approximations, J. Funct. Anal. 259 (3) (2010), 561-582.

[17] P. Hájek and M. Johanis, Smooth Analysis in Banach Spaces, Series in Nonlinear Analysis and Applications, De Gruyter, 2014.

[18] M. Jiménez-Sevilla and L. Sánchez-González, LFC bumps on separable Banach spaces, J. Math. Anal. Appl. 365 (1) (2010), 315-319.

[19] M. Jiménez-Sevilla and L. Sánchez-González, Smooth extension of functions on a certain class of non-separable Banach spaces, J. Math. Anal. Appl. 378 (1) (2011), 173-183.

[20] M. Jiménez-Sevilla and L. Sánchez-González, On smooth extensions of vector-valued functions defined on closed subsets of Banach spaces, Math. Ann. 355 (2013), 1201-1219.

[21] N.J. Kalton and G. Lancien, Best constants for Lipschitz embeddings of metric spaces into $c_{0}$, Fund. Mat. 3 (2008), $249-272$.

[22] S. Keith, A differentiable structure for metric measure spaces, Adv. Math. 183 (2) (2004), 271-315. 
[23] B. Kleiner and J. Mackay, Differentiable structures on metric measures spaces: a primer, Ann. Sc. Norm. Super. Pisa Cl. Sci. 16 (5) (2016), no. 1, 41-64.

[24] J.R. Lee and A. Naor, Extending Lipschitz functions via random metric partitions, Invent. Math. 160 (2005), 59-95.

[25] J. Pechanec, J.H.M. Whitfield and V. Zizler, Norms locally dependent on finitely many coordinates, An. Acad. Brasil. Ciênc. 53 (3) (1981), 415-417.

[26] J. Pelant, Embeddings into c c, Topology Appl. 57 (1994), 259-269.

[27] L. Sánchez-González, On smooth approximation and extension on Banach spaces and applications to Banach-Finsler manifolds, PhD. Dissertation, Departamento de Analisis Matematico, Facultad de Matematicas, Universidad Complutense de Madrid, 2012.

[28] H. Toruńczyk, Smooth partitions of unity on some non-separable Banach spaces, Studia Math. 46 (1973), 43-51.

[29] H. Whitney, Analytic extensions of differentiable functions defined on closed sets, Trans. Amer. Math. Soc. 36 (1) (1934), 63-87.

[30] H. Whitney, Differentiable functions defined in closed sets I, Trans. Amer. Math. Soc. 36 (2) (1934), 369-387. 